\title{
On a Possible Mechanism of Quiescent Prominence Destabilization
}

\section{P. Nenovski}

Geophysical Institute, Acad. G. Bonchev Str, 1113 Sofia, Bulgaria

V. N. Dermendjiev and M. S. Madjarska

Institute of Astronomy, 72 Trakia Blvd., 1784 Sofia, Bulgaria

J.-C. Vial

\section{I.A.S., Université Paris XI-CNRS, Bâtiment 121, 91405 Orsay, France}

\begin{abstract}
The possibility of multiple reflections generating standing surface wave modes carrying field-aligned current (FAC) in an arch-like prominence structure is studied. The conditions for enhancement of FAC leading to observable destabilising effects are specified.
\end{abstract}

\section{Introduction}

In this work we study the possibility that some surface MHD modes, generated in a simple arch-shaped prominence structure, can enhance the electric current along the magnetic field, named the field-aligned current (FAC). We try to derive a criterion determining the commencement of the prominence instability.

\section{MHD Surface Wave Model of FAC Intensification}

First attempts to construct a model of FAC carried by MHD surface modes were made by Nenovski and Momchilov (1987). Madjarska et al. (1996) studied a surface wave FAC intensification process relevant to quiescent prominences (QP). It was shown that nonaxial surface waves (SW) propagating obliquely to the ambient magnetic field carry FAC. Such an MHD surface mode "induces" an inertial term in the corresponding equation for the FAC. These FACs are located at the prominence boundaries.

According to Madjarska et al. (1996) the FAC intensification depends on the reflection coefficient, $\mathrm{R}$, for SW modes in the prominence feet and the SW dissipation in the prominence body. The necessary condition for FAC enhancement in a prominence, with arch length $L$, can be given by the inequality

$$
(L(A) / L) \ln (1-R)>1 .
$$

FAC can be intensified when both the reflection coefficient $R<0$ and the FAC gain exceeds the SW dissipation. We express the SW dissipation by the characteristic damping length $L(A)$. 


\subsection{Reflection Conditions in the Prominence Feet}

We examine a simple model of a prominence foot depending only on the $\mathrm{z}^{-}$ coordinate, perpendicular to the solar limb. The calculation grid has a step $\Delta \mathrm{z}$ $=200 \mathrm{~km}$ and knots number $\mathrm{N}_{z}=10$. This corresponds to a $2000 \mathrm{~km}$ high prominence foot. The magnetic field is computed using the formula $B=B_{0} \exp ^{-2 \pi z / L}$, where $B_{0}$ is a constant. Here we take two types of initial conditions: 1) plasma parameters similar to those of Hvar Reference Atmosphere of QP (Engvold et al. 1990) and 2) plasma parameters corresponding to the VAL network chromosphere model (Vernazza et al. 1981), i.e., supposing a plasma upflow in the prominence feet. The corresponding reflection coefficient $R$ is given by

$$
R=\frac{\sum_{A}-\sum_{p}}{\sum_{A}+\sum_{p}}
$$

where $\sum_{p}=\int \sigma_{\perp} d z$ is the height-integrated Pedersen conductivity, and $\sum_{A}=$ $1 / \mu v_{A}$ is the Alfven wave conductivity. $\mathrm{R}$ is derived under the condition $\operatorname{div} \vec{j}=0$, which means that the FAC will be completely closed by the perpendicular Pedersen and Hall current flowing in the prominence feet at the prominence-chromosphere system. The perpendicular to the magnetic field conductivity $\sigma_{\perp}=\sigma_{\perp, i}+\sigma_{\perp, e}$ is computed taking into account the ion-neutrals, $\nu_{i n}$, and electron-neutrals, $\nu_{e n}$, collision frequencies under the assumption for pure Hydrogen plasma.

Setting the first initial condition in the computation procedure, we obtain $R$ positive. However, under the second initial condition we obtain $R$ negative, even $R=-1$. This should lead to FAC intensification at each reflection event.

In the computations of $\sum_{p}$, at each step we verify the inequality $\nu_{i n}>\omega_{c i}$, where $\omega_{c i}$ is the cyclotron-ion frequency. The equality $\nu_{i n}=\omega_{c i}$ marks the maximum height of the conductivity layers, where the FAC could be closed. In Figure 1 (top) the map represents the dependence of the quantity $\left(\nu_{i n}-\omega_{c i}\right)$ on Hydrogen concentration $n_{H}$ and magnetic field $B$. The reflection coefficient $R$, computed for 5 different values of $B$ is given in Figure 1 (bottom) as a function of the height (z-coordinate). The basic conclusion is that $R$ will be negative (reflection of SW in the prominence feet will occur) when the plasma density increases in the prominence feet, and/or when the axial component of the ambient magnetic field is strongly weakened.

\subsection{SW Dissipation in the QP Body}

The intensification process of the surface wave FAC depends on the ratio $L(A) / L$. This means that the damping length $L(A)$ has to be large enough than $\mathrm{L}$, so that the $\mathrm{SW}$ will be multiply reflected. We suppose that in prominence conditions the viscous losses dominate and we can use for the damping length the formula

$$
L(A)=\left\langle s_{z}\right\rangle / Q,
$$

where $Q$ is the volumetric heating rate, and $\left\langle s_{z}\right\rangle$ is the time-averaged z-component (along the FAC direction) of the Poynting flux.

When $\omega_{c i}>\nu_{i n}$ (this condition is fulfilled in the prominence body)

$$
Q=\frac{P}{\nu_{\text {in }}}(\nabla \cdot \vec{v})^{2} / 3
$$




$$
\left\langle s_{z}\right\rangle=\rho_{0}\left[c_{0}^{2}+v_{A}^{2}\right](\nabla \cdot \vec{v})^{2} k_{\perp} / \omega^{3},
$$

hence

$$
L(A)=\rho_{0} L^{3} \omega_{c i} /(2 \pi)^{2} t_{A} \rho_{n},
$$

where $c_{0}$ is the sound velocity, $\omega$ is the SW frequency, $P$ is the pressure, $k_{\perp}$ is the normal component of the wave vector. It is clear that the damping length is sensitive to the SW period, $t_{A}$, and the neutral density, $\rho_{n}$.

\section{Criteria for Destabilization of a Loop-Shaped QP}

Starting from the inequality (1) we can obtain the following criteria for destabilization. When $R=-1$, then $\ln (1-R)=0.7$ and we obtain

$$
(L(A) / L)>1.4 \text {. }
$$

From the ion collision damping condition $\nu_{i n}<\omega_{c i}$ we obtain

$$
\frac{n_{H}}{B}<a_{1} \times 10^{23} \text { (in SI units), }
$$

where $a_{1}$ is a constant of order of $0(1)$. Additional restrictions on $n_{H}$ and magnetic field variation we obtain from the condition of the closure of the FAC

$$
n_{H} \sqrt{n} / B k_{\perp}<1.5 \times 10^{32} \text { (in SI units), }
$$

The derived relationships $(6,7,8$ and 9$)$ give all principal requirements for the intensification of FAC by standing $\mathrm{SW}$ and the possible consequent $\mathrm{QP}$ destabilization, and allow a direct observational verification even in the case when the magnetic field in the prominence body remains unknown.

\section{Conclusion}

The inclusion of surface MHD modes as FAC carriers in a model of QP destabilization seems to have a good perspective. However, some details of the model need additional examination by means of numerical simulations.

Acknowledgments. One of the authors (V.N.D.) is very grateful to the Organizing Committee of the IAU Colloquium 167 for funding his participation. This work was partially supported by the National Scientific Foundation (Bulgaria) under Grant ST-618/96.

\section{References}

Engvold, O., Hirayama, T., Leroy, J.-L., Priest, E. R. and Tandberg-Hanssen, E. 1990, in Dynamics of Quiescent Prominences, V. Ruždjak and E. Tandberg-Hanssen (eds.), Springer-Verlag, New York, p. 294

Madjarska, M. S., Dermendjiev, V. N., Mouradian, Z. and Nenovski, P. 1996, Astroph. Lett. and Comm, 34, 113

Nenovski P., Momchilov, G. 1987, Planet. Space Sci., 35, 1561

Vernazza, J., Avrett, E., Loeser, R. 1981, ApJS, 45, 635 

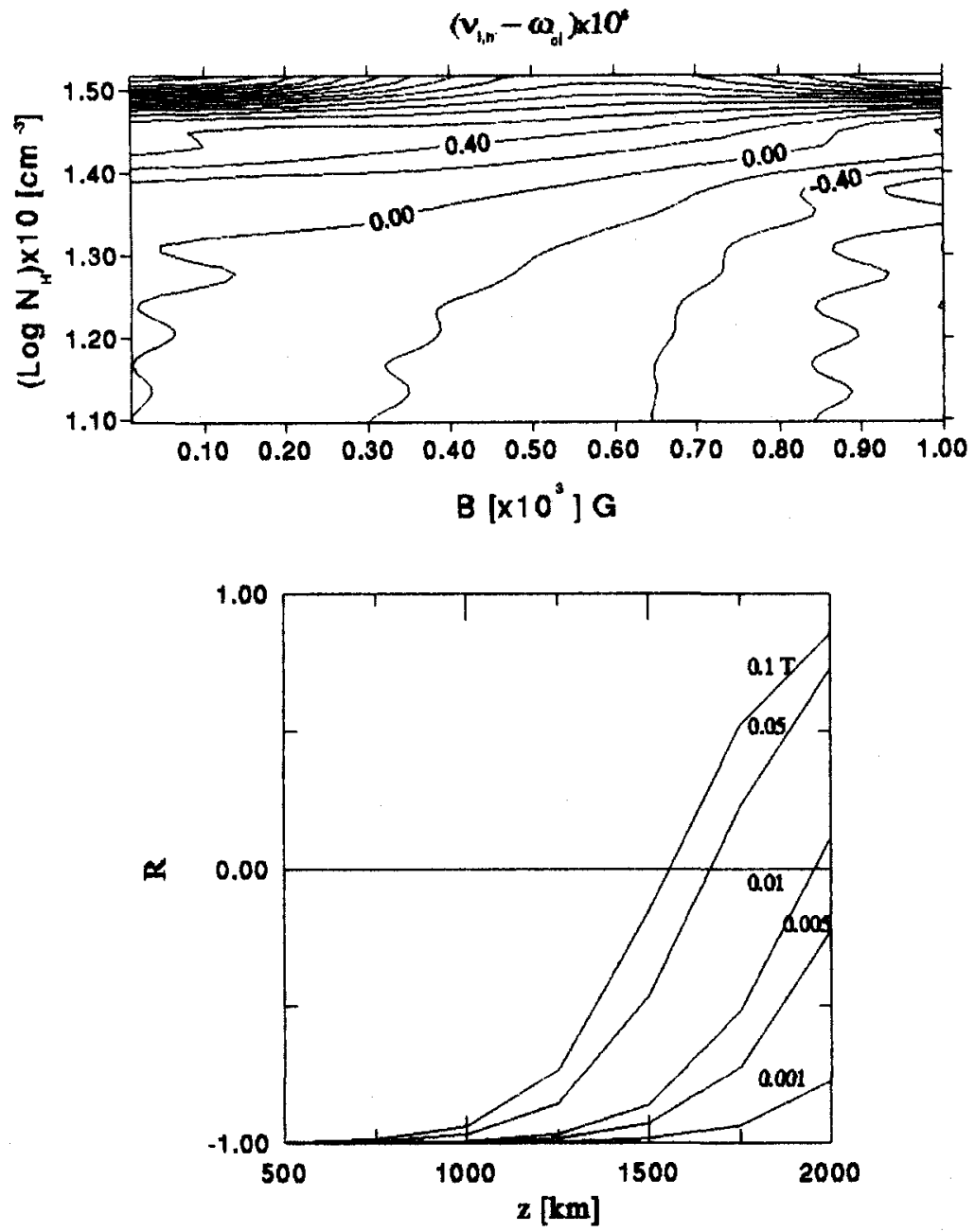

Figure 1. Top: Isopleth map representing the quantity $\left(\nu_{i n}-\omega_{c i}\right)$ as a function of the $\mathrm{H}$-concentration, $N$, and the magnetic induction, $B$. Bottom: The reflection coefficient, $R$, as a function of the height, $z$, for given values of the magnetic induction. 